\title{
A study of functional outcomes of distal humerus fractures operated by triceps reflecting approach
}

\author{
Sarukte $V^{1}$, Bhanushali $R^{2}$, Nagrale $U^{3}$, Vishwakarma $S^{4}$ \\ ${ }^{1}$ Dr Vijay Sarukte, ${ }^{2}$ Dr. Ravi Bhanushali, ${ }^{3}$ Dr. Umesh Nagrale, ${ }^{4}$ Dr. Sunil Vishwakarma, all authors are affiliated with \\ Department of Orthopedics, K. B. Bhabha Hospital Bandra (w), India.
}

Address for Correspondence: Dr Vijay Sarukte, E-mail: vlsarukte@rediffmail.com

\begin{abstract}
Introduction: Approaches to distal humerus has been a great controversy of which cheverons osteotomy is considered a gold standard. However it is associated with implant and procedure related complication, Hence we studied a triceps reflecting (Bryan -Morrey) approach with some modification so as to achieve good exposure to distal humerus with intact olecranon. Methods: In our study we retrospectively studied 13 skeletally mature distal humerus fractures at our center treated by TRA with minimum follow up for a period of 3 months. They were functionally assessed with DASH Score, MAYO score, triceps power evaluation with MRC grading, measurements of ROM and Radiological union. Results: Mean duration of follow up was Ten months in 13 patients, with an average age of 43.23 years. The mean Quick DASH score was 3.85, mean Mayo score was 89 indicating an good performance, mean Triceps muscle power MRC grading 5 with adequate mean functional ROM 23-114 degree(extension -flexion). Only 2 patients had complication of which one was screw breakage and other was wound dehiscence which was not related to approach. Conclusion: TRA is a better option as it avoids creating a new fracture and preserving the joint anatomy as compared to olecranon osteotomy with adequate exposure of the joint to carry out the fixation. As from our series functional outcomes of strength of triceps is comparable with that of the other side.
\end{abstract}

Keywords: DASH Score, MAYO score, MRC grade, triceps reflecting approach, Bryan Morrey approach, distal Humerus fractures.

\section{Introduction}

Intra-articular fractures of the distal humerus constitute $0.5 \%-7 \%$ of all fractures and $30 \%$ of elbow fractures. These fracture in the younger age groups are secondary to high energy trauma and in elderly women as a result of relatively low energy trauma [1]. The treatment of intra-articular distal humerus fractures is matter of continuous debate in the orthopedic literature. They are uncommon, the anatomy is complex, and bone is frequently comminuted. It explains why these fractures pose a significant challenge for the orthopedic surgeon. [2]. Preferred treatment for displaced, intra-articular, intercondylar fractures of the distal part of the humerus is open reduction and internal fixation. Adequate exposure of the articular surface of the distal humerus and elbow joint is required for operative stabilization of

Manuscript received: $15^{\text {th }}$ January 2017

Reviewed: $22^{\text {nd }}$ January 2017

Author Corrected: $28^{\text {th }}$ January 2017

Accepted for Publication: $6^{\text {th }}$ February 2017 bicolumnar distal humerus fractures [3]. Although the posterior approach using the olecranon chevron osteotomy is considered the gold standard, the reconstruction of the osteotomy may lead to complications that include delayed union, wound dehiscence, nonunion, malunion, hardware failure, and pain secondary to prominent hardware. Alternative approaches to avoid these complications have been reported during the last years, such as the tricepssplitting, triceps-reflecting anconeus pedicle, the anconeus flap transolecranon approach, and the tricepssparing approach [2].

This all approaches avoid an osteotomy and mobilize the triceps and anconeus muscle off the posterior humerus and the intermuscular septae and provides adequate exposure for open reduction and internal fixation. The median exposed articular surface for the 
triceps splitting, triceps reflecting, and olecranon osteotomy approaches was 35\%, 46\%, and 57\%, respectively. Olecranon osteotomy exposed more articular surface than the triceps splitting approach (Mann-Whitney test, $\mathrm{P}=.03$ ) but was not significantly greater than the triceps reflecting approach. However, even the olecranon osteotomy approach failed to provide visualization of more than $40 \%$ of the distal humeral articular surface [4,5]. The purpose of our study was to determine the functional outcome of fixation of displaced intra-articular distal humeral fractures with use of triceps reflecting Bryan-Morrey approach.

\section{Material and Methods}

Our study was performed at secondary care municipal hospital in Mumbai from 12/03/2014 to 11/06/2015. Inclusion criteria was 13 fresh close distal Humerus Fractures, presenting to our OPD/ Emergency Room. Exclusion criteria was pathologic fractures from primary or metastatic tumors, comminuted, no associated systemic injuries and age under 18 years. All patients were subjected to radiographs of the involved elbow in AP and lateral views with CT-Scan of the elbow. Fractures were classified as per AO type A to C (except C3 comminuted) and were included in this prospective study in whom internal fixation was done using triceps reflecting Bryan Morrey approach with some modification in 4 cases. Basic patient demographics, mechanism of injury and AO fracture classification were recorded. The functional outcome of patients was assessed using MAYO and Quick DASH (Disability of arm, shoulder and hand) scoring system with strength of triceps using MRC Grades [6, 7]. Postoperative radiographs were reviewed for evidence of bony union or complications (non-union, avascular necrosis, implant failure, etc.).

This information was entered into a Microsoft Excel database for statistical analysis. After medical evaluation and pre-anesthetic check-up, informed written consent was taken from patient and were taken up for surgery. Patients were operated under general anesthesia or supraclavicular and axillary block in lateral decubitus position. The advantages of this position include ease of access to the posterior elbow for fracture fixation, without the need for extra assistants. Patients were operated under upper arm pneumatic tourniquet, with routine deflation after $2 \mathrm{~h}$ for procedures that exceed this length of time.
Surgical Approach- Generally, intra-articular fractures of the distal humerus is accessed by the posterior approach, which gives excellent exposure of the articular fragments of the distal humerus. This approach requires reflection of the extensor mechanism, typically through either a triceps splitting approach or an olecranon osteotomy or a triceps reflecting approach. The transolecranon exposure for distal humerus fractures is a very popular technique that is suggested for improving articular visualization and allowing accurate reduction.

There are several modifications to this technique, such as the chevron shaped olecranon osteotomy, commonly advocated by the AO group. The chevron osteotomy increases rotational and translational stability at the time of surgery and increases the contact area for achieving the bony union. It has the advantage of same exposure of articular surface of distal humerus without disturbing the articular surface of olecranon, thereby reducing the chances of elbow stiffness and improving the range of movements postoperatively. To avoid complications, it is strongly advisable that the osteotomy should be fixed by tension band wiring with two tightening loops. Alternatively, a pre-contoured olecranon plate may be used. Significant osteotomy complications have prompted recommendations for alternative exposure techniques. Distally, intra-articular exposure is dependent on triceps mobilization, and there are many modifications in the posterior elbow surgical approaches. These are triceps splitting at midline, triceps reflecting van Gorder or Bryan Morrey, triceps reflecting anconeus pedicle (TRAP), anconeus flap trans-olecranon (AFT), and para-tricipital approaches.

In our study, we are using triceps reflecting Bryan Morrey approach for intra-articular exposure and fixation of fracture distal humerus with some modification in 4 selected cases.

In this procedure, the extensor mechanism comprising the triceps tendon, forearm fascia, and periosteum are reflected as one unit from the medial to lateral off the olecranon i.e. thin bone sleeve is lifted. In some cases triceps tendon was split into two and only medial or the lateral triceps tendon sleeve with bony chip was reflected. The ulnar nerve is first identified and protected. A periosteal elevator is used to dissect the triceps muscle from the posterior humeral cortex. With a scalpel, the forearm fascia, periosteum, and triceps tendon are reflected directly off the olecranon from medial to lateral as a continuous sleeve. The triceps 
may be removed along with a thin wafer of bone to facilitate bone to bone rather than tendon to bone healing at the triceps insertion site. Now the entire triceps muscle with the posterior capsule is reflected upwards and laterally, and the elbow is flexed to expose the joint. At the end of the procedure the triceps tendon is reinserted back on to the olecranon by means of nonabsorbable sutures passed through transosseous drill holes in the olecranon. The triceps repair needs protection for 4-6 weeks postoperatively hence following the operation, the elbow was immobilized in a splint at $90^{\circ}$ of flexion. The arm was kept elevated for 3-4 days in order to reduce the edema and inflammation.

The splint was subsequently removed and active assisted ROM exercises were initiated after suture removal. Ice was applied following the exercises. On average the patients were discharged at the end of the first week. At this point the splint was removed and a hinged elbow brace was used to protect the internal fixation. Indomethacin prophylaxis for heterotopic ossification was given for 10 days. Patient were advised to avoid active elbow extension against resistance to prevent triceps suture failure. Patients were followed-up on a weekly basis for the first six weeks. Then the patients were seen every month for follow-up until the 3rd postoperative month.

Surgical Technique-The position of patient during surgery varies according to the complexity of the fracture. The patient is positioned in the lateral decubitus position on a bolster or beanbag, with the entire upper extremity draped free and the surgery was performed with the use of a tourniquet. The shoulder was placed at $90^{\circ}$ flexion and the elbow at $90^{\circ}$ flexion. A midline straight skin incision was made with the proximal two-thirds of the incision above the tip of the olecranon while the remaining one-third was over the back of the forearm from the tip of the olecranon.

The distal part facilitates exposure and isolation of the ulnar nerve which is critical for the safe exposure of the distal humerus. After exposure and isolation of the ulnar nerve, the scalpel was used to sharply separate the anterio-medial border of the triceps muscle from the medial intermuscular septum down to the bone. Sharp dissection down to the bone was also done laterally between the antero-lateral border of the triceps muscle and the lateral intermuscular septum with the radial nerve and the profunda brachii artery passed within it from the back of the arm anteriorly, so that the back of the humerus could be safely reached without endangering these vital structures. By elevation and retraction of the whole bulk of the muscle, the posterior surface of the humerus could be safely reached without interruption or violation of the integrity of the triceps muscle and its tendon. The distal humerus articular surface is reassembled and the intra-articular components of the fracture are reduced first and then temporarily fixed using Kirschner wires. Once the joint surface has been restored and reconstructed anatomically, the metaphyseo-diaphyseal fragments are reduced and again temporarily fixed with K-wires, stabilizing both the columns.

The metaphyseal butterfly, if present, is also anatomically reduced to maintain the length of column. When there is inter-condylar comminution or bone loss/bone missing from the trochlea, care should be taken not to narrow the trochlea. This may require placement of a piece of structural graft, usually obtained from the iliac crest. This was fixed with $4.5 \mathrm{~mm}$ partially threaded inter-condylar screw. Once satisfactory anatomical reconstruction of the distal humerus has been achieved, then final fixation of the distal humerus is done.

The anatomical plates (combi hole-LC-DCP, recon and $1 / 3$ tubular plate) were placed to the medial and lateral columns in 90-90 configuration after molding to their respective shape. For the fixation of medial column $1 / 3^{\text {rd }}$ tubular was preferred and was placed medially as per the medial column contour while lateral plate was placed on posterior surface of the lateral column. The less comminuted column was fixed initially and then the other column was fixed. Plate length was chosen according to the proximal extension of the fracture line and each plate was fixed at least with three bi-cortical screws at the diaphysis. The plates were fixed to the distal fragment with $3.5 \mathrm{~mm}$ cortical screws, extending to the opposite condyle and the proximal fixation was initially done with a cortical screw. Then the fracture was compressed at the supracondylar level with the insertion of an eccentric screw through one of the proximal holes in both plates.

An attempt was made to hold the distal fragments together by multiple locking screws as much as possible extending to the opposite column. Proximal fragments were fixed according to the configuration of the fracture by at least three bicortical screws. 


\section{Results}

Mean duration of follow-up was Ten months (range 3 months-18 months) in 13 patients, with Mean age of 43.23 years (range 25-70 years). 6 were female, and 7 were male, of which 2 female were young and 4 were in older age group (above 50 years) while all 7 males were young.

Mean tourniquet time was $104 \mathrm{~min}$ (range 70 to $130 \mathrm{~min}$ ). According to the AO Classification there were Two A2, Three B1, four C1, three C2 and one each B2 and A3. Strength of the triceps muscle (power) was assessed by using MRC grading. The mean strength of the injured side for extension was FIVE, the mean strength for flexion was FIVE and was comparable to other side.

The mean Quick DASH score was 3.85 (range, 0-11.4). The mean Mayo score was 89 (range 75-100), indicating an excellent performance. 9 patients $(75 \%)$ had an excellent outcome while 4 (25\%) patients had good outcome.

Mean Range of motion in our study was 23-114 degree, with only two cases had worse range of motion. Mean flexion in our study was 114 degree, Range 90 to 140 (normal range120-145degree) with Mean flexion deficit compared to other uninjured side 25 degree range 0 to 50 degree. Mean extension in our study was up to 23 degree, range 10 to 40 degree (normal 5 to -15 degree) with extension deficit of 28 , range 15 to 45 degree compared to other side.

No patient achieved complete extension while only two patient achieved complete flexion comparable to other uninjured side. Arc of motion was 91 degree. No limitation in the pronation-supination was detected.

Radiographic assessment Postoperative and follow up radiographs showed adequate fracture reduction and fracture healing in all patients. No step off more than one mm was seen. Radiologic follow up of at least 3 months postoperative (mean ten months) revealed in one cases visible osseous chip on the tip of the olecranon, one patients had radiologic signs of posttraumatic arthritis at follow up X-ray at 14 months.

One patient had infection which recovered with post-operative antibiotic coverage. one patients had transient ulnar nerve palsies that had recovered completely at the last follow-up.one patient had wound problem and implant was visible which required longer time for immobilization and reoperation with one column plate removal and skin grafting (Mean ROM 40 to 100, MRC grade three, Quick DASH 9.1 and Mayo 75) and one patient had intra - articular lag screw breakage restricting her range of motion to 40 to 90 degree, Quick DASH score 11.4 and Mayo score 75 as shown in figure 1.

Overall complication was seen in $15 \%$ of the patients $(n=2)$. patient with stiffness were advised for arthrolysis for further ROM but were not willing for procedure.
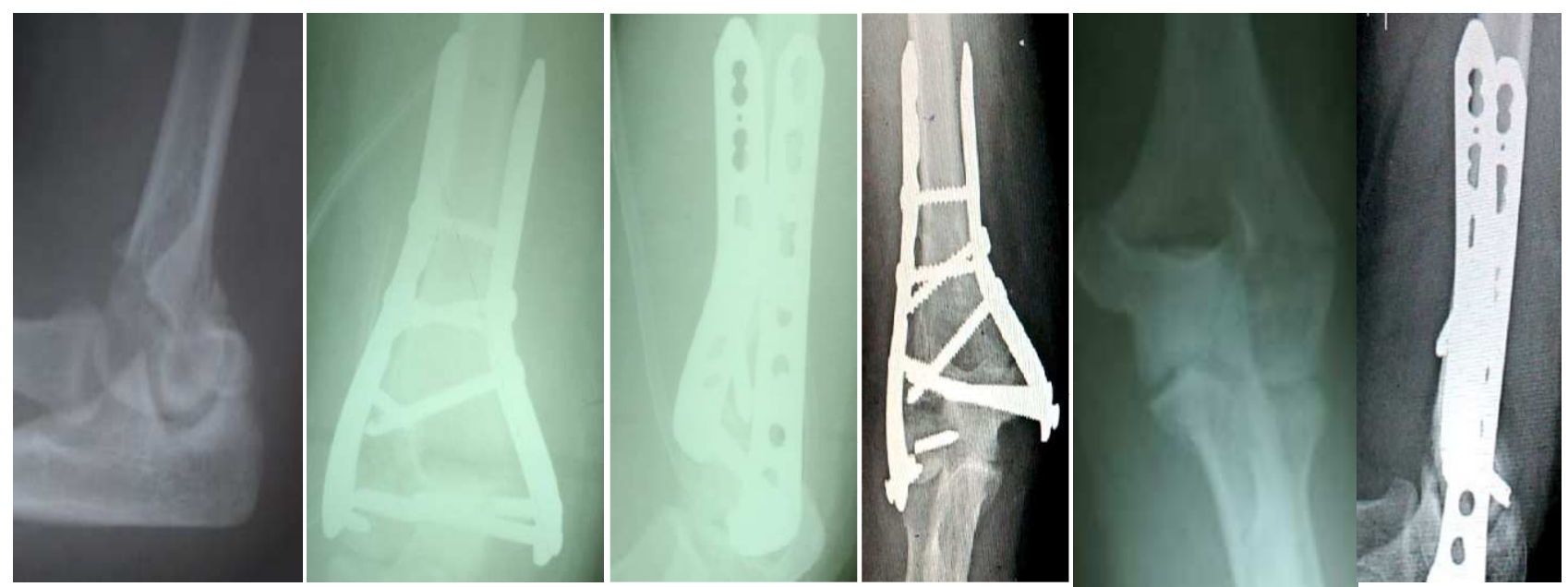

Figure No-1: AP and LAT pre-operative and post-operative with screw breakage at final follow up 

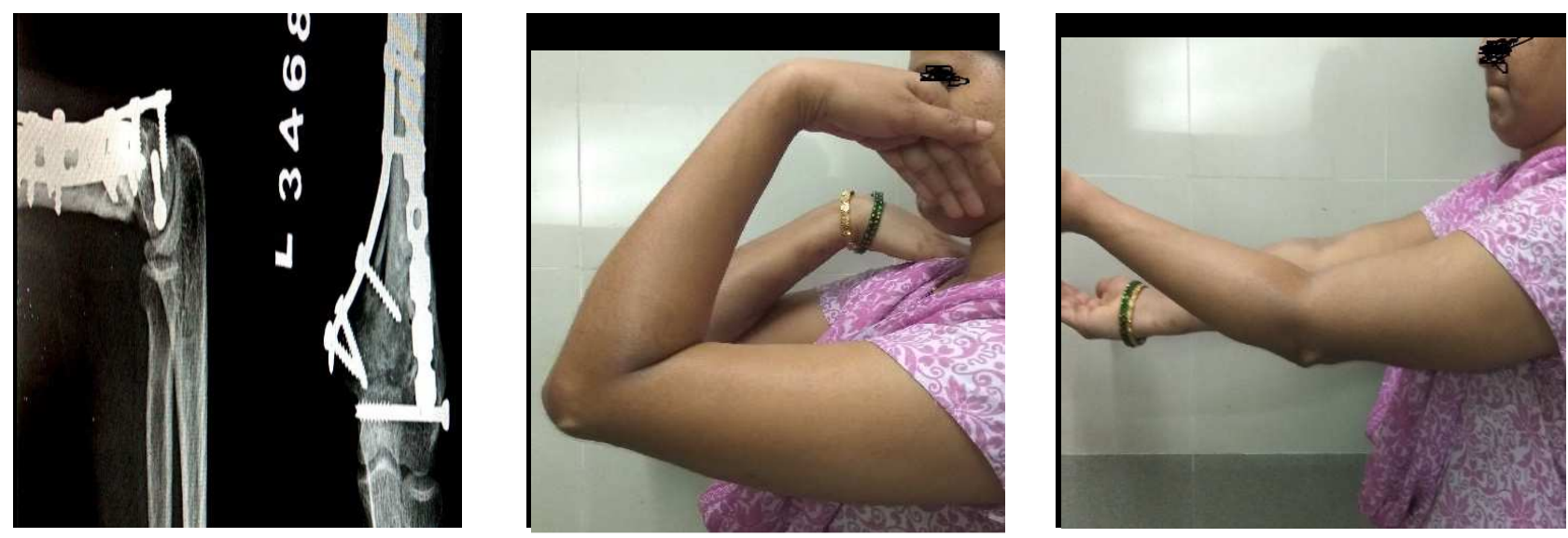

Figure-2: Post operative radiographs, functional ROM Flexion -Extension

\section{Discussion}

Surgery in distal humerus fractures is difficult, because of the complex anatomy, small size of the fractured fragments and poor bone quality; nevertheless, with careful preoperative planning, surgical expertise, stable fixation and with early aggressive postoperative mobilization, functional results are satisfactory in $75 \%$ to $85 \%$ of cases [8]. For fracture fixation most commonly used approaches are olecranon osteotomy, triceps splitting (Campbell), triceps dividing (TRAP, Van Gorder), triceps sparing and triceps reflecting (Bryan Morrey approach). Each approach has its own indications and contra indications. Surgeon's preference is one of the important factor in deciding the surgical approach. So, the surgical approach that not only provides the surgeon adequate visualization of the distal part of the humerus and the articular surface, but also minimizes disruption of the soft tissue and elbow extensor mechanism is chosen. Traditionally, the olecranon osteotomy approach has been considered gold standard for distal humeral exposure, however this exposure has additional potential complications, like delayed union, olecranon nonunion, risk of ulnar nerve injury, implant-related issues, and implant prominence. Coles et al in his study showed that $8 \%$ of patients operated by olecranon osteotomy required hardware removal [9]. Tak et al showed that their osteotomies united in an average of 11 weeks (range, 8-20 weeks) with no non-unions but 4 delayed unions, which all healed by 20 weeks without any intervention.

Their most frequent complication was symptomatic osteotomy fixation in $19 \%$, all of them needed removal of the implant after the osteotomy had united. Symptomatic olecranon fixation has a $71 \%$ correlation with non-satisfactory results [10]. Schmidt-Horlohe et al. reported a series of 31 patients with type-C-fractures of the distal humerus treated by ORIF via olecranon osteotomy and re-fixation of the osteotomy with hook plates. In this series removal of the hook plate was performed in $48.4 \%$ of patients [11].

The other triceps dividing approaches like Triceps reflecting anconeus pedicle (TRAP) approach and Van Gorder approach are becoming popular approaches. In these approaches the entire triceps anconeus pedicle flap is reflected proximally after releasing the triceps muscle from olecranon. The main drawback of this approach is triceps dehiscence and extensor weakness. Another triceps dividing approach used is the Triceps Tongue approach, (Van Gorder) in which the triceps tendon is divided at its musculo-tendinous junction. In this technique, the transection of the triceps is done in form of "V". This approach also carries the same risks and complications as described for TRAP approach. Campbell described yet another approach for distal humerus fractures in which midline incision was taken at the triceps through triceps tendon. Partial excision of olecranon tip was done for better visualization. Limited visibility, triceps dehiscence, extensor mechanism dehiscence were some of the drawbacks of this approach [12].

The triceps reflecting approach, as described by Bryan and Morrey, exposes $46 \%$ of the articular surface which is $11 \%$ less than osteotomy, but this difference was not found to be statistically significant. This was shown by Wilkinson and Stanley who demonstrated in a cadaver model study that the proportion of articular surface exposed in the triceps splitting, triceps reflecting, and olecranon osteotomy approaches was $35 \%, 46 \%$, and 
$57 \%$, respectively [4]. Similar information is provided by Dakoure'et al. with 26, 37 and 52\% exposure [13]. Further, triceps elevating exposures can be associated with weakness of extension or even rupture of the triceps, although a recent study of central triceps peel showed similar extension strength as in patients undergoing olecranon osteotomy. Triceps splitting approach can also cause triceps weakness due to resultant fibrosis and injury to intermuscular nerve branches [14]. But the dissection by Bryan Morrey and TRAP approaches are in the inter-nervous plane, the above-mentioned problems do not occur. Hence triceps reflecting approach is gaining popularity which prevents complications of osteotomy. The Bryan and Morrey medial approach to the elbow involves raising the triceps and extensor mechanism as a full sub-periosteal sleeve off of the posterior humerus and olecranon. This requires meticulous technique so as not to violate the continuity of the extensor mechanism during dissection. Reflection of the sleeve of extensor mechanism results in exposure of the elbow joint. Closure is performed by returning the extensor mechanism to its appropriate position along the posterior tip of olecranon and repair with non-absorbable sutures and trans-osseous tunnels. But the approach has been associated with extensor mechanism weakness or failure and ulnar nerve dysfunction [15].

Our technique allows the reflecting of the major part of the triceps in continuity with its attachment at the olecranon. Protection of the ulnar nerve by the medial part of triceps reduces the possibility of damage to its blood supply and at the end of the operation it can glide and slide in its original position. The availability of the two segments of the triceps muscle for the repair allows satisfactory balancing of the medial and lateral sides of the elbow, reducing the risk of postoperative dislocation.

The hemi-peel (partial tendon bone sleeve) modification of the approach represents a compromise between the excellent articular surface visualization provided by trans-olecranon osteotomy and triceps reflecting approaches and the extensor mechanism preservation of the para-tricipital approaches. The hemi-peel approach attempts to mitigate the risk of extensor mechanism weakness and ulnar nerve injury by limiting dissection to the lateral $50 \%$ of the triceps periosteal insertion on the proximal ulna i.e. olecranon. It exploits the greater laxity of the lateral structures of the elbow as a window for visualization of the articular surface. No osteotomy or hardware is required, and the medial insertion of the triceps is maintained. In our study of 13 cases, hemi peel was used in 4 cases while in remaining 9 cases full periosteal sleeve was removed for better exposure of articular surface of distal humerus. This modification of triceps hemi-peel was studied by Grogan et al by TRIFCU approach [16].

Triceps strength function is also better recovered in patients operated by Bryan - Morrey approach and this is reflected in Mayo elbow score which is still in favor of this approach. Another advantage of Brayan - Morrey approach is that it exempt from the complications of the olecranon osteosynthesis material or its migration. Given the issues described above, for type B and C fractures of the distal humerus, Bryan - Morrey approach should be considered as a better option. The potential complications of Bryan-Morrey's approach include triceps avulsion and triceps weakness. This exposure has also been widely used predominantly for elbow arthroplasty and has been criticized for weakness in extension postoperatively; other complications such as infection, reoperation or loss of strength are rare. Guerroudj et al. compared the in vitro mechanical properties of the triceps tendon after simulation of three common exposures and showed that all approaches resulted in a weakening of the triceps; however, the Bryan-Morrey lateral triceps-reflecting technique provided statistically better strength than V-Y or longitudinal splitting [17]. There are only few articles in the literature reporting on the use of a triceps sparing approach to the distal humerus in trauma. Ek et al in their report of 9 adolescents showed an average triceps deficit compared with the uninvolved arm of 6-10\% with this approach [18]. So they proposed the BryanMorrey triceps-sparing approach as a safe option for Tcondylar distal humeral fractures in adolescents.

Postoperative radiographs show anatomic reconstruction of the joint surface and of the extraarticular angles in all cases, thus indicating that the exposure provided by the triceps reflecting approach was adequate. Moreover, an intact olecranon can serve as a template for reconstruction of the trochlea, especially in osteoporotic bone at the risk of narrowing the trochlear width by compression due to poor bony resistance. Furthermore, closure is comparably quicker. By reducing the reflected extensor apparatus back in place and fixing the bony chip with a figure of eight non-resorb able trans osseous suture, closure is achieved faster and is technically less challenging than 
olecranon osteotomy approach. The strength measurements revealed no statistically significant loss of function of the extensor apparatus on the injured side, on objective and subjective scores i.e. Quick DASH and Mayo Elbow Performance Score. The ROM was in functional range to carry out activities of daily living. Askew et al reported loss of strength of triceps in all patients with olecranon osteotomy or triceps splitting approach. The operative fixation performed in our cases showed stable enough fixation for postoperative rehabilitation. Our main concern was avoidance of any postoperative extensor apparatus dysfunction. None of the cases operated in our study resulted in any dysfunction at elbow or showed other mechanical or neurological deficit.

Morrey et al. in study of 15 activities of daily living with respect to elbow motion and forearm rotation in a normal elbow, showed that $100^{\circ}$ of elbow flexion and $100^{\circ}$ of forearm rotation are required for most of the activities of daily living [19]. Vasen et al also studied 12 activities of daily living in one hundred elbows in normal population with respect to flexion and extension [20]. By isolating the allowable ROM of the elbow and allowing for compensatory motions and strategies of the normal adjacent joints, the functional elbow. ROM was established as $75-120^{\circ}$ flexion. In our study, the mean elbow flexion was $114^{\circ}$, the mean forearm extension was $23^{\circ}$ and the mean arc of motion was $91^{\circ}$ which is within the functional elbow ROM. Our results are comparable to other published studies $[18,21]$.

In our study as most complex fractures were addressed, four patients eventually had some limitation to the range of motion. However, the functional scores of these patients were satisfactory. Despite the mild to moderate limitation of motion, these patients could continue with their daily life. In another longer term (19 years) study by Doornberg et al, a similar result has been reported [22].

We therefore conclude that the triceps reflecting approach to treat distal intra-articular humerus fractures does not lead to functional disadvantages. Operated cases may suffer from post-traumatic arthritis of the elbow later in life. Hence, a longer follow-up will be required. In conclusion, this triceps reflecting approach provides an excellent exposure as well as a good functional outcome as quantified by DASH Score without any dysfunction of extensor apparatus of elbow.
Short duration of follow up and relatively small sample size is the limitation of this study. A further study with larger sample size and longer follow up will be required to provide proper guidelines.

\section{Conclusion}

Triceps reflecting Bryan Morrey approach is a simple and effective approach that can be used in management of the majority of the distal humeral fractures as it provides adequate visualization and reduction fracture fragments with no adverse effect on triceps muscle strength.

\section{Funding: Nil, Conflict of interest: None. Permission of IRB: Yes}

\section{Bibliography}

1. Babhulkar S, Babhulkar S. Controversies in the management of intra-articular fractures of distal humerus in adults. Indian J Orthop. 2011 May; 45 (3): 216-25. doi: 10.4103/0019-5413.80039.

2. Fernández-Valencia J, Muñoz-Mahamud E, Ballesteros J, Prat S. Treatment of AO Type C Fractures of the Distal Part of the Humerus through the BryanMorrey Triceps-Sparing Approach. ISRN Orthopedics. 2013; 2013:1-6.

3. Pankaj A, Mallinath G, Malhotra R, Bhan S. Surgical management of intercondylar fractures of the humerus using triceps reflecting anconeus pedicle (TRAP) approach. Indian Journal of Orthopaedics. 2007; 41 (3):219.

4. Wilkinson JStanley D. Posterior surgical approaches to the elbow: A comparative anatomic study. Journal of Shoulder and Elbow Surgery. 2001; 10 (4): 380382 .

5. Azboy I. The comparison of triceps-reflecting anconeus pedicle and olecranon osteotomy approaches in the treatment of intercondylar fractures of the humerus. Turkish Journal of Trauma and Emergency Surgery. 2015.

6. Mayo Elbow Performance Score. Journal of Orthopaedic Trauma. 2006; 20 (Supplement):S127.

7. Hudak P, Amadio P, Bombardier C, Beaton D, Cole

$\mathrm{D}$, Davis A et al. Development of an upper extremity 
outcome measure: The DASH (disabilities of the arm, shoulder, and head). American Journal of Industrial Medicine. 1996; 29(6):602-608.

8. Henley MB, Bone LB, Parker B. Operative management of intra-articular fractures of the distal humerus. J Orthop Trauma. 1987;1(1):24-35.

9. Coles C, Barei D, Nork S, Taitsman L, Hanel D, Bradford Henley M. The Olecranon Osteotomy: A Sixyear Experience in the Treatment of Intraarticular Fractures of the Distal Humerus. Journal of Orthopaedic Trauma. 2006;20(3):163-170.

10. Tak SR, Dar GN, Halwai MA, Kangoo KA, Mir BA. Outcome of olecranon osteotomy in the transolecranon approach of intra-articular fractures of the distal humerus. Ulus Travma Acil Cerrahi Derg. 2009 Nov;15(6):565-70.

11. Schmidt-Horlohé K, Wilde P, Bonk A, Becker L, Hoffmann R. One-third tubular-hook-plate osteosynthesis for olecranon osteotomies in distal humerus type-C fractures: a preliminary report of results and complications. Injury. 2012 Mar;43(3):295300. doi:10.1016/j.injury.2011.06.418.Epub 2011Jul 22.

12. Bucholz RW. Rockwood and Green's fractures in adults: Lippincott Williams \& Wilkins Philadelphia; 2006.

13. Coulibaly NF, Tiemdjo H, Sane AD, Sarr YF, Ndiaye A, Seye S. Posterior approach for surgical treatment of neglected elbow dislocation. Orthop Traumatol Surg Res. 2012 Sep; 98(5):552-8. doi: 10. 1016/j. otsr.2012.03.006. Epub 2012 Aug 1.

14. Kasser J, Richards K, Millis M. The TricepsDividing Approach to Open Reduction of Complex Distal Humeral Fractures in Adolescents: A Cybex Evaluation of Triceps Function and Motion. Journal of Pediatric Orthopaedics. 1990;10(1):93-96.
15. Bryan RS, Morrey BF. Extensive posterior exposure of the elbow. A triceps-sparing approach. Clin Orthop Relat Res. 1982 Jun; (166):188-92.

16. Grogan BF, Blair JA, Blease RE, Cho MS, Hsu JR. Exposure of the distal humerus using a triceps hemipeel approach. Orthopedics. 2014 May;37(5):e455-9. doi: 10.3928/01477447-20140430-56.

17. Guerroudj M, de Longueville J, Rooze M, Hinsenkamp M, Feipel V, Schuind F. Biomechanical properties of triceps brachii tendon after in vitro simulation of different posterior surgical approaches. Journal of Shoulder and Elbow Surgery. 2007;16 (6): 849-853. doi: 10.1016/j.jse.2007.02.137.

18. Ek E, Goldwasser M, Bonomo A. Functional outcome of complex intercondylar fractures of the distal humerus treated through a triceps-sparing approach. Journal of Shoulder and Elbow Surgery. 2008;17(3): 441-446. doi: 10.1016/j.jse.2007.08.012.

19. Morrey BF, Askew LJ, Chao EY. A biomechanical study of normal functional elbow motion. J Bone Joint Surg Am. 1981 Jul;63(6):872-7.

20. Vasen AP, Lacey SH, Keith MW, Shaffer JW. Functional range of motion of the elbow. J Hand Surg Am. 1995 Mar;20(2):288-92.

21. Zhang C, Zhong B, Luo CF. Comparing approaches to expose type $\mathrm{C}$ fractures of the distal humerus for ORIF in elderly patients: six years clinical experience with both the triceps-sparing approach and olecranon osteotomy. Arch Orthop Trauma Surg. 2014 Jun; 134(6):803-11. doi: 10.1007/s00402-014-1983-y. Epub 2014 Apr 29.

22. Doornberg JN, van Duijn PJ, Linzel D, Ring DC, Zurakowski D, Marti RK, Kloen P. Surgical treatment of intra-articular fractures of the distal part of the humerus. Functional outcome after twelve to thirty years. J Bone Joint Surg Am. 2007 Jul;89(7):1524-32.

\section{How to cite this article?}

Sarukte V, Bhanushali R, Nagrale U, Vishwakarma S. A study of functional outcomes of distal humerus fractures operated by triceps reflecting approach. Int J Med Res Rev 2017;5(02):105-112. doi:10.17511/ijmrr. 2017.i02.02. 